\title{
STUDIES WITH A NEW METHOD FOR DETERMINING THE COAGULATION TIME OF THE BLOOD \\ IN THE NEW-BORN *
}

\author{
F. C. RODDA, M.D. \\ Assistant Professor of Pediatrics, University of Minnesota \\ CHILDREN'S CLINIC OF MINNEAPOLIS
}

In pursuance of studies on the coagulation time of blood in the new-born clinic of the pediatric department, University of Minnesota, there was found a host of methods from which to choose, none of which were particularly adapted to the work or gave dependable results.

Since Vierordt's early studies on blood coagulation many ingenious devices and methods have been employed. Hinman and Sladen ${ }^{1}$ gave a history and résumé of the work. The more practicable of these methods fall into certain types, and these were studied.

First, was considered Wright's ${ }^{2}$ method and its many modifications. These, however, call for the use of special, carefully calibrated tubes with very close temperature control. Even so, results vary widely. Another type of method was introduced by Milian ${ }^{3}$ and modified by Duke, Hinman and Sladen. ${ }^{1}$ This method, however, gives marked variations according to the quantity of blood deposited on the slide, and Duke's ${ }^{4}$ technic requires a specially constructed slide, hard to obtain. Brodie and Russell's ${ }^{5}$ instruments, with modification by Boggs, present another scheme. These instruments, however, are special, expensive, require the use of a microscope and are not practicable for general bedside use. Besides, the method employs a current of air directed against the drop. Differences of humidity, temperature and force of the air current introduce factors which affect the results and are hard to control.

Howell criticized all methods employing blood obtained by skin puncture because of the mixture of tissue juices which' shorten the

* Read before the Northwestern Pediatric Society.

1. Hinman, F., and Sladen, F. J.: Measurements of the Coagulation Time of the Blood and Its Application, Johns Hopkins Hosp. Bull. 18:207, 1907.

2. Wright, A. E.: On a Method of Determining the Condition of Blood Coagulability for Clinical and Experimental Purposes, Brit. M. J. 2:223, 1893.

3. Milian, G.: Le Causes d'erreur dans l'etude Clinique de la Coagulabilete' du sang, Presse méd. 1:202, 1904.

4. Duke, W. W.: A Simple Instrument for Determining the Coagulation Time of the Blood, Arch. Int. Med. 9:258 March) 1912.

5. Brodie, T. G., and Russell, A. E.: The Determination of the Coagulation Time of the Blood, J. Physiol. 21:403, 1897. 
time. His method, as modified by Lee and White, ${ }^{6}$ in which the blood is obtained by venipuncture and the clotting time obtained in a small glass tube, is the most often employed. Doubtless, it is the method which, when carefully followed, comes nearest to giving the absolute coagulation time. However, the venipuncture must be done skillfully, with minimal injury to the vessel wall, and the blood must be obtained without the admission of air bubbles, or widely varying results occur. Also, the end result is not sharply defined.

In work on the new-born one is at once confronted with the difficulty of obtaining blood from a superficial vein. It is extremely difficult, often impossible. It is true, one can enter the superior longitudinal sinus, but this requires a special technic. Even the most experienced often fail to enter the sinus because of distortion from molding of the head. It is especially difficult in the first days of life, and besides, the procedure of thrusting a needle through the scalp into the cranial cavity is not always innocuous. It surely is no routine to be practiced by a novice in the home.

A method was, therefore, sought which would require little special apparatus and be simple, easily applied in the home by any physician engaged in the care of the new-born. A return was made to the drop method. All factors such as depth and site of puncture, size and sequence of the drop, temperature, air currents, and the effect of foreign bodies in contact with the blood, were duly considered. An effort was made to control and standardize all these factors and still have the procedure simple and easy of application in the ward or the home. After many trials the following method was evolved.

\section{METHOD}

The apparatus required consists of a spring lance (a simple scalpel will suffice), two one and one-half inch watch glasses and No. 6 lead shot, all of which are easily obtained and transported. Glass and shot should be cleaned, preferably by washing with soap and water followed by alcohol and ether. Needless to state, the lance should be sterile, which implies freedom from old blood. The heel of the infant is sponged with ether, a puncture is made with the lance blade set (about 0.5 c.m.) to produce a free flow of blood without the slightest pressure. A clean watch glass containing a No. 6 shot receives the second drop of blood. A second watch glass is inverted over the first. The watch glasses are gently tilted every thirty seconds until the shot no longer rolls, but is fixed in the clot. The end-result is sharply defined, the shot is firmly embedded, so that the glass may be inverted without dislodgment of the shot. At times, due to the forcing out of

6. Lee, R. I., and White, P. D.: A Clinical Study of the Coagulation Time of Blood, Am. J. M. Sc. 145:495, 1913. 
serum, the whole clot may move, carrying the shot with it. This is no source of confusion, for the shot will be found enmeshed in the fibrin and there is no rolling or rotation of the shot. A poorly formed clot or one showing slight retraction is patent, for in such instances the shot continues to roll.

An effort was made to comprehend and check all sources of error. Coating the shot with paraffin did not affect the time. Watch glasses of the same size and curve were employed to standardize the surface area of the drop in contact with glass. The inverted watch glass minimizes the drying of the drop and prevents the entrance of lint and dust. All the work was done in the new-born ward, and the slight variations of the room temperature did not affect results. The greatest source of error is concerned with the blood flow. A small, slowly forming drop obtained by pressure clots very quickly, the tenth drop more quickly than the second. Depth and extent of the cut show no influence as long as the flow of blood is free and occurs without

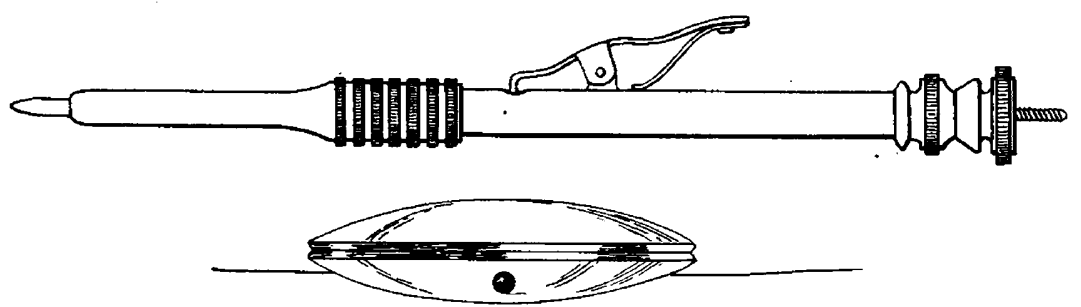

Fig. 1.-The apparatus used consists of a spring lance, two watch crystals and No. 6 lead shot.

pressure or squeezing. In all the determinations the blood flow was free. The first drop was discarded, the second drop, only, employed, and time was reckoned from the moment that the first drop fell. The time consumed in the formation of the second drop was considered a part of the coagulation time. Determinations were made to the nearest half minute.

No claim is made that the absolute clotting time is obtained, but the result is, at least, a clean cut relative time for comparative work. The object is to find gross variations from a normal range. The question is not whether the clot forms in six minutes or in six minutes and forty-five seconds, but whether it forms in six or sixteen minutes.

This method was checked with that of Lee and White in a series of thirty-eight determinations done on the same infants at the same time, blood being obtained for the Lee and White method from the superior longitudinal sinus. The average time was the same-seven minutes-for both methods. This was quite unexpected, for it has been assumed that all methods employing skin puncture give shorter 


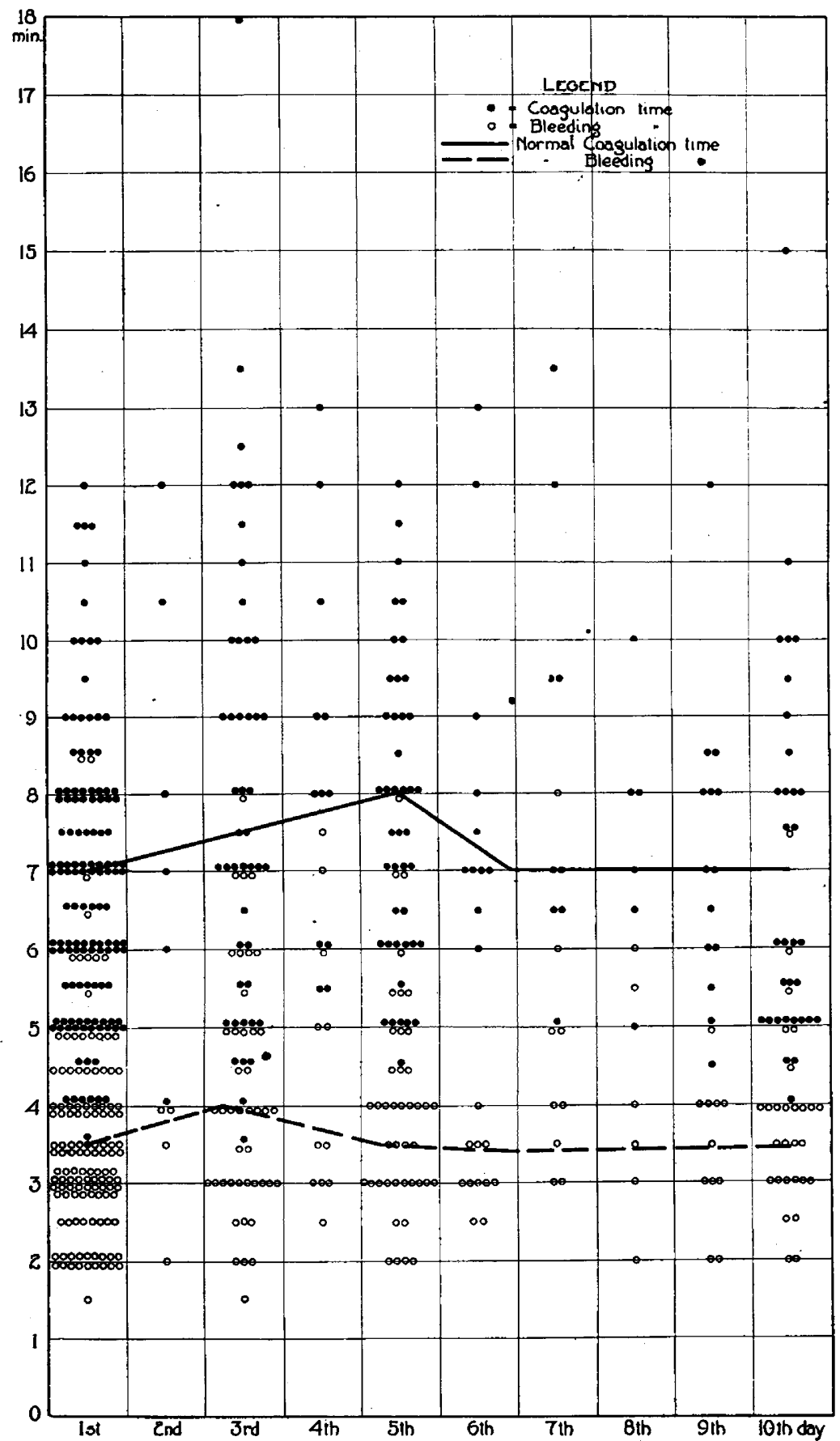

Fig. 2.-Determinations of coagulation and bleeding time on various days of first ten. 


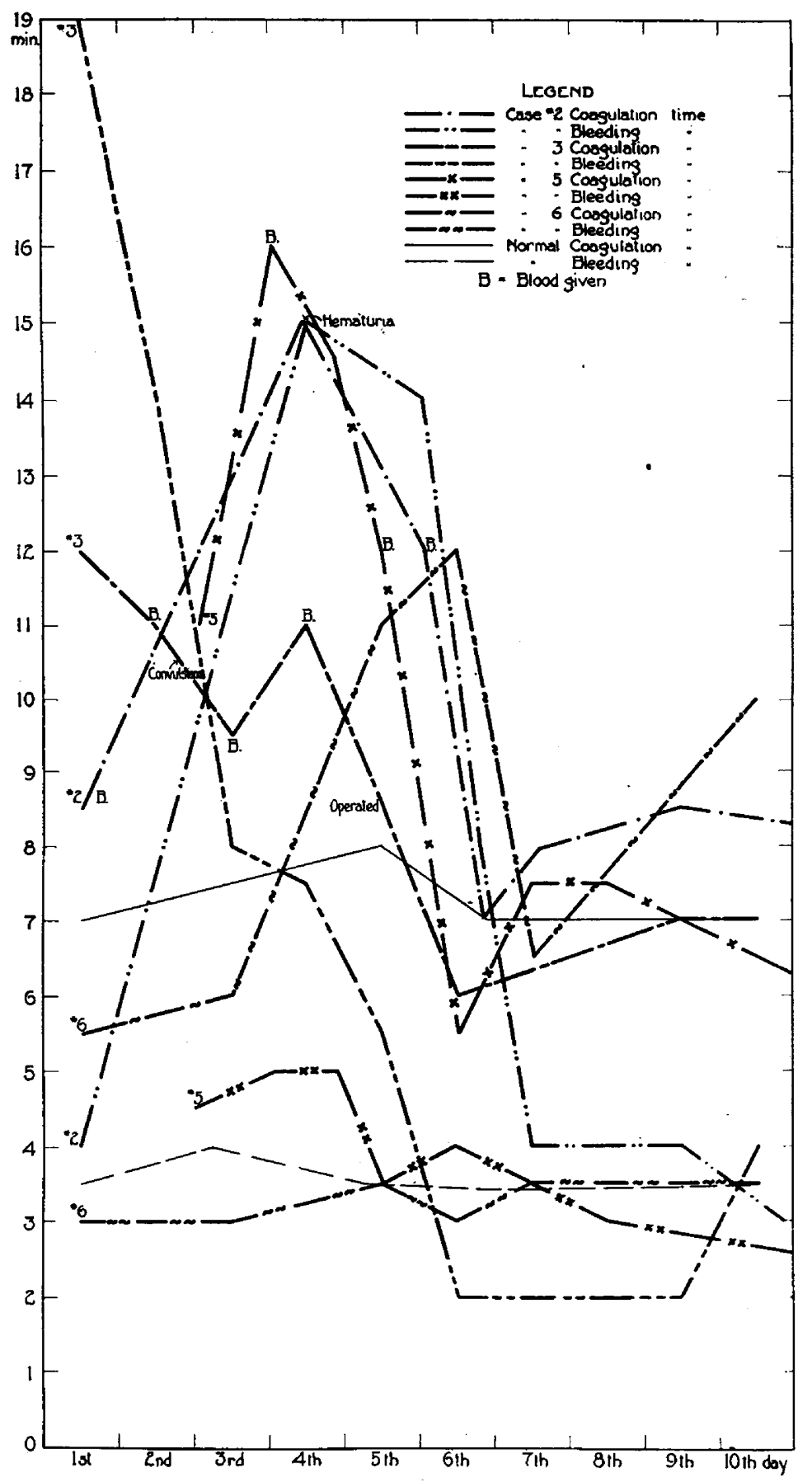

Fig. 3.-Coagulation time in a series of cases above the normal range. 
clotting times. Further, the Lee and White method showed a greater variation than the drop method. In the series with the Lee and White method the arithmetical deviation from the average of seven minutes was two and one-half minutes, or 36 per cent., while the drop method gave a variation of one and one-half minutes from the average of seven minutes, or 20 per cent. In the same series the standard deviation with the Lee and White method was approximately three and one-half minutes, and with the drop method two minutes. In a small series of adults and older infants and children the results with the method coincided with those obtained in the new-born.

The average coagulation time determined on 126 new-borns within the first twenty-four hours of life was seven minutes with an arithmetical deviation of one and one-half minutes, giving an approximate range of from 'five and one-half to eight and one-half minutes, or roughly, from five to nine minutes. Eighty per cent. of all determinations fall within this range, while a range of from four to ten minutes included 95 per cent. of all the cases. It may, then, be stated that with this method the average coagulation time is seven minutes-that a time of more than ten minutes presents delayed coagulation.

It was early noted that the coagulation time varied on different days of the infant's life, showing a tendency to prolongation over the second, third and fourth days with a maximum on the fifth day, and a return to the time obtained during the first twenty-four hours before the tenth day.

Determinations with the method were then made on various days of the first ten, the majority being taken on the first, third, fifth and tenth days. A field graph and a median curve of the results were prepared. Because of the smaller number of determinations on the second, fourth and sixth days, combinations of second and third, fourth and fifth, sixth and seventh, eighth and ninth days were made. The result is then a weighted curve of the median. It is to be noted that the curve reaches the highest points on the fourth and fifth days. It is significant that this corresponds with the age incidence of hemorrhage disease in the new-born.

The bleeding time, using Duke's ${ }^{7}$ method, was also determined, and a similar weighted curve constructed. It shows the same general type of curve as that of the clotting time. The average time in the same 126 cases was three and one-half minutes, with an average deviation of one and one-half minutes, and a range from two to five minutes. A bleeding time of from two to five minutes may be considered normal for the new-born.

7. Duke, W. W.: The Relation of Blood Platelets to Hemorrhagic Disease, J. A. M. A. 55:1185 (Oct. 1) 1910. 
A study was then made of a series of cases presenting coagulation times above the normal range. In this chart the curves of the average normal coagulation and bleeding times are shown, also four abnormal ones with case numbers. It is to be noted, that all these cases presented an exaggeration of the normal curve of coagulation time. It is further to be noted that in two cases a prolonged bleeding time accompanied the delayed coagulation time. In Cases 2 and 3 hemorrhages appeared. In Case 2 a marked hematuria occurred on the fourth day. Blood was given subcutaneously, the reactions returned to normal and the hematuria cleared up. In Case 3 convulsions developed, and other signs of cerebral hemorrhage, on the second day. Blood was given subcutaneously; a decompression operation was performed on the fifth day. Recovery was complete.

On the other hand, cases showing prolonged coagulation time, accompanied by a normal bleeding time, showed no signs of hemorrhage (Cases 5 and 6). It would appear, therefore, that the hemorrhagic factor is a complex of delayed coagulation time and prolonged bleeding time. Further, variations from the normal range of coagulation and bleeding times are due to some deficiency of coagulation properties or excess of anticlotting substances which may gradually be overcome. This may be hastened by the subcutaneous administration of blood (Case 2). In all the cases a return to normal finding occurred by the tenth day. Further studies on calcium, prothrombin, "antithrombin," and fibrinogen content of the new-born blood are under way.

\section{SUMMARY}

1. The method is simple, easily applied in the new-born and gave results more accurate than those obtained with the Lee and White method.

2. The average coagulation time in the new-born with the method is seven minutes, with a normal range of from five to nine or ten minutes. The average bleeding time in the new-born with Duke's method is three and one-half minutes with a normal range of from two to five minutes.

3. In the normal new-born there is found a tendency to prolongation of these times in the first days of life. Hemorrhage is accompanied, in many cases at least, with an exaggeration of this tendency or markedly prolonged coagulation and bleeding times.

4. The coagulation and bleeding times should be studied in conjunction.

5. Routine determinations in the new-born will allow the selection of at least certain of the hemorrhagic conditions before the onset of symptoms and give the indication for the administration of blood. 
276 AMERICAN JOURNAL OF DISEASES OF CHILDREN

6. The method will allow of a check on blood therapy in hemorrhages of the new-born.

7. Further studies may help to clarify the complex known as hemorrhagic disease of the new-born.

The writer is indebted to Prof. R. E. Scammon of the Department of Anatomy for valuable suggestions and help in preparing the field graphs and curves. 